\title{
Clinical case of portal vein thrombosis at the liver cirrhosis patient
}

\begin{abstract}
We present clinical case of portal vein thrombosis at the liver cirrhosis patient with signs of severe decompensation of liver function and portal hypertension. A feature of this case is that after consumption of alcohol condition of the patient was estimated erroneously as alcoholic hepatitis with high Maddrey index (105). However, later diagnosis of portal vein thrombosis was confirmed. The cancellation of antiinflammatory therapy and administration of anticoagulants allowed to stabilizate of critical situation. Thus, the clinical experience has shown that dopplersonography is necessary at the liver cirrhosis, especially in case of decompensation.
\end{abstract}

Keywords: liver cirrhosis, portal vein thrombosis, portal hypertension, hepatic failure, dopplersonography, vitamin $\mathrm{K}$ antagonists, low molecular weight heparins
Volume 9 Issue 6 - 2018

\author{
Ermolova T,' Bykova E,' Petrov A² \\ 'North-Western State Medical University named after II. \\ Mechnikov, St-Petersburg, Russia \\ ${ }^{2}$ City Hospital 2, St.-Petersburg, Russian Federation
}

\begin{abstract}
Correspondence: Ermolova Tatiana PhD,Associate Professor at the Department of Faculty Therapy, FSBEl North-Western state medical university, named after I.I Mechnicov of RMN, StPetersburg,Email t.v.ermalova@mail.ru
\end{abstract}

Received: August 30, 2018 | Published: November 12, 2018

\section{Introduction}

the diagnosis of portal vein thrombosis (PVT) was first diagnosed in 1862 by Botkin SP. in Russia and in Europe it was first described in 1868 by Balfour GW and Stewart TG. ${ }^{1,2}$ The thrombosis were considered unlikely at the liver cirrhosis and anticoagulants were contraindicated, but modern studies have shown that PVT is not uncommon. Prevalence of PVT ranges from $2.1 \%$ to $23.3 \%$ among candidates for liver transplantation without HCC. ${ }^{3}$ According to autopsy, the frequency of PVT in liver cirrhosis is 6-64\%. ${ }^{4}$ Clinical symptoms range from asymptomatic to acute rapid thrombosis

\section{Case report}

Patient B., female, 52yrs. Anamnesis: alcohol abuse is for many years, she noted changes in liver tests in during 10yrs. Diagnosis of the alcoholic steatohepatitis, cirrhotic stage, class A (Child-TurcottePugh score) was confirmed 6years ago. She took periodically various drugs - ursodeoxycholic acid, silimarin. In during the holiday in Spain she felt satisfactory, consumed about 30-40grs of alcohol per day. After the arrival in a week patient noted the gradual appearance of weakness, nausea, repeated vomiting, then moderate jaundice and itching of the skin, drowsiness, moderate enlargement of abdomen, legs edema. She was hospitalized in the Gastroenterology department of St.-Petersburg City Hospital 2 and patient's condition was severe. The condition was regarded as "Alcoholic liver disease: steatohepatitis, high activity (acute alcoholic hepatitis?). Cirrhotic stage, class C of Child-Turcotte-Pugh score, MELD-25, Maddrey Index-105. Syndrome of Portal hypertension (splenomegaly, hypersplenism, ascites, enlarged esophageal veins 1 degree) and liver failure (1 stage of hepatic encephalopathy, decreased prothrombine, albumin, cholesterol)". Autoimmune, viral screening, ceruloplasmin were normal. The doctors recommended anti-inflammatory treatment (prednisolone 30mgs), diuretics (spironolactone 100mgs), therapy of hepatic encephalopathy (rifaximin, lactulose, Hepa-Merz), betablockers. Despite the ongoing therapy, the her condition worsened in the form of an increase in hepatic insufficiency and cholestasis: decrease of blood total protein from 60 to $52 \mathrm{~g} / 1$, albumin to $29 \mathrm{~g} / \mathrm{l}$, cholesterol from 2.1 to $1.07 \mathrm{mmol} / 1$, platelets - to $66 \times 10^{9} / \mathrm{L}$, increase of ALT from 183 to $188 \mathrm{U} / \mathrm{l}$, AST from 311 to $333 \mathrm{U} / \mathrm{l}$, total bilirubin from $22.1 \mathrm{mg} / \mathrm{dl}$ (direct-12.7mg/dl) to $27.4 \mathrm{mg} / \mathrm{dl}$ (direct-17,6mg/dl). We noted normalization of electrolytes and decrease of ascite. Hepatic encephalopathy $1^{\text {st }}$ stage progressed to encephalopathy $2^{\text {nd }}$ stage.
The consultant-hepatologist recommended an US dopplerography that detected signs of portal vein thrombosis. Conclusion of USdopplerography: portal hypertension, occlusive PVT with not determined blood flow, passabled vena cava inferior (diameter $17.5 \mathrm{~mm}$, collapsing to $50 \%$-normal), decreased monophonic blood flow in hepatic veins. Enlarged spleen vein to $13 \mathrm{~mm}$ with inverted blood flow and enlarged hepatic artery to $5 \mathrm{~mm}$.

Patient was consulted by hepatosurgeon: risk of surgical treatment is very high due to severe condition of patient and contraindications for operation. Hepatologist stopped prednisolone and it was decided to prescribe anticoagulants according to international recommendations. ${ }^{5,6}$ We started a treatment with Xa factor selective inhibitor - Natrium Fondaparinux (Arixtra) $5 \mathrm{mg}$, then $2 \mathrm{mgs}$ per day s.c. , Rabeprazole. In some days we noted a considerable positive dynamics of clinical and laboratory data with gradually improvement of hepatic function, decrease of cholestasis and cytolysis in next weeks. Control US-Dopplerography revealed positive dynamics: appearance of initial recanalization of portal vein, mosaic blood flow. In 2 weeks control triplex US dopplerography revealed signs of partial recanalization of portal vein to $45 \%$ with linear parietal blood flow and linear speed of blood flow $8 \mathrm{sm} / \mathrm{sec}$. Spleen vein decreased to $7,0 \mathrm{~mm}$, cava inferior vein non enlarged, $12 \mathrm{~mm}$, phased blood flow.

The patient discharged from the hospital in satisfactory condition. We recommended the introduction of the Arixtra 6weeks under the supervision of a physician and the control of clinical, biochemical analysis, coagulogram, US-dopplerography. In future it's necessary to decide the question of administration of indirect anticoagulant (AVK). It is recommended to continue taking of rabeprazole, creon, lactulose, Hepa-Merz, spironolactone, beta-blockers. Conclusion: this Case Report demonstrated, that at the chronic liver diseases patients with cirrhotic stage it's necessary to administer Ultrasound Dopplerography, especially with decompensation, for diagnosis of the portal vein thrombosis.

\section{Acknowledgements}

None.

\section{Conflict of interest}

The author declares no conflict of interest. 


\section{References}

1. Polovtsov AA. Russian biographic dictionary. 1908;3:710.

2. Balfour GW, Stewart TG. Case of enlarged spleen complicated with ascites, both depending upon varicose dilatation and thrombosis of the portal vein. Edinb Med J. 1869;14(7):589-598.

3. Rodriguez-Castro KI, Porte RJ, Nadal E, et al. Management of non neoplastic portal vein thrombosis in the setting of liver transplantation: a systematic review. Transplantation. 2012;94(11):1145-1153.
4. Bakulin IG, Shalikiani NV. Trombosis of portal vein: modern sight to questions of ethiopathogenesis, prevention and treatment. Effective pharmacotherapy. Gastroenterology. 2014;2(43):64-69.

5. EASL Clinical Practice Guidelines: Vascular diseases of the liver. Journal of Hepatology. 2016;64(11):179-202.

6. Delgado MG, Seijo S, Yepes I, et al. Efficacy and Safety of Anticoagulation on Patients With Cirrhosis and Portal Vein Thrombosis. Clin Gastroenterol Hepatol. 2012;10(7):776-783. 